TRANSACTIONS OF THE

AMERICAN MATHEMATICAL SOCIETY

Volume 358, Number 2, Pages 491-507

S 0002-9947(05)04084-5

Article electronically published on September 26, 2005

\title{
CONSTANT MEAN CURVATURE SURFACES IN $M^{2} \times \mathbf{R}$
}

\author{
DAVID HOFFMAN, JORGE H. S. DE LIRA, AND HAROLD ROSENBERG
}

AbStRaCt. The subject of this paper is properly embedded $H$-surfaces in Riemannian three manifolds of the form $M^{2} \times \mathbf{R}$, where $M^{2}$ is a complete Riemannian surface. When $M^{2}=\mathbf{R}^{2}$, we are in the classical domain of $H$-surfaces in $\mathbf{R}^{3}$. In general, we will make some assumptions about $M^{2}$ in order to prove stronger results, or to show the effects of curvature bounds in $M^{2}$ on the behavior of $H$-surfaces in $M^{2} \times \mathbf{R}$.

\section{INTRODUCTION}

There is an enormous difference between the theory of minimal surfaces $(H=0)$ and nonzero constant-mean curvatures surfaces $(H \neq 0)$, and this is most evident in $\mathbf{R}^{3}$. A properly embedded minimal surface in $\mathbf{R}^{3}$ cannot be compact; if it is simply connected, it must be the plane or the helicoid; and if it is an annulus, it must be the catenoid 15, 3. For $\Sigma$, a properly embedded surface of nonzero constant mean curvature in $\mathbf{R}^{3}$ : if $\Sigma$ is compact, it must be a round sphere; if $\Sigma$ is noncompact, it cannot be simply connected; and, if $\Sigma \sim S^{1} \times \mathbf{R}$, it must be rotationally symmetric (a Delaunay surface). For both $H=0$ and $H \neq 0$, there are many known examples of finite topology with genus greater than zero and more than two ends, all discovered in the last twenty years 6, 7, 27, 26, 28].

The theory of minimal surfaces in $M^{2} \times \mathbf{R}$ is now well developed from the point of view of examples and theory [17, 21, 16]. However, the theory of $H$-surfaces in $M^{2} \times \mathbf{R}$ is just beginning. (We will assume throughout, unless we say so explicitly, that $H \neq 0$.) With one exception, there are no general theorems in the literature, but there is useful information in some special cases. The exception is when $M^{2}=$ $T^{2}$ with the flat metric. Here we are considering doubly periodic $H$-surfaces in $\mathbf{R}^{3}$, and there are existence results that come from the theory of triply periodic $H$-surfaces in $\mathbf{R}^{3}$ [9, 10, 5]. There are many known examples. Additional examples come from the existence of doubly periodic $H$-surfaces in $\mathbf{R}^{3}$ [12.

When $M^{2}=S^{2}$, there is a class of examples due to Hsiang and Hsiang, 8 , and Pedrosa and Ritoré, 20], who studied the isoperimetric problem in $S^{n} \times \mathbf{R}$. Among

Received by the editors December 1, 2003.

2000 Mathematics Subject Classification. Primary 53C27, 58J60.

The first author was partially supported by research grant DE-FG03-95ER25250/A007 of the Applied Mathematical Science subprogram of the Office of Energy Research, U.S. Department of Energy, and National Science Foundation, Division of Mathematical Sciences research grant DMS-0139410.

The second author was partially supported by Cooperaçao Brasil-França - Ministère des Affaires ètrangères (France) and CAPES (Brasil). 
other things, they analyzed the rotationally-invariant $H$-surfaces in $S^{n} \times \mathbf{R}$. Let

$$
B=\frac{S^{n} \times \mathbf{R}}{O(n)}=\{(x, y) \mid x \in[0, \pi], y \in \mathbf{R}\},
$$

and consider the generating curves $\gamma(s)=(x(s), y(s))$ satisfying

$$
\gamma^{\prime}(s)=(\cos \sigma, \sin \sigma),
$$

where $\sigma=\sigma(s)$ satisfies

$$
\frac{d \sigma}{d s}=H+(n-1) \cot (x) \sin (\sigma) .
$$

Their $O(n)$-orbits are $H$-surfaces. Pedrosa and Ritoré found first integrals and solutions of Delaunay-type, i.e. properly embedded and periodic $H$-hypersurfaces that were annular (topologically $S^{n-1} \times \mathbf{R}$ ). They also found closed profile curves that give rise to compact tori (topologically $S^{1} \times S^{n-1}$ ) that are unstable, and bounded profile curves that define examples that are hyperspheres.

A motivating question for this paper is the following: Are there properly embedded annular $H$-surfaces in $S^{2} \times \mathbf{R}$ that are not of Delaunay type (i.e., rotational)? Korevaar, Kusner and Solomon proved that, in $\mathbf{R}^{3}$, all such surfaces are Delaunay 11 .

The authors thank Rick Schoen for useful conversations concerning the rescaling argument used in the proof of Theorem 3 in Section 5.

\section{Height estimates in $M^{2} \times \mathbf{R}$}

For an interval, $I \subset \mathbf{R}$, we define $M_{I}:=M^{2} \times I \subset M^{2} \times \mathbf{R}$. When $I=\{a\}$, we will write $M_{a}^{2}=M^{2} \times\{a\}$.

We prove height estimates for compact embedded $H$-surfaces $\Sigma$ with boundary in some $M_{a}^{2}$. The estimates depend on curvature bounds for $M^{2}$ and on the value of $H$. We also prove that when $M^{2}$ is compact and $\Sigma$ is a noncompact and embedded $H$-surface, that $\Sigma$ has both a top and a bottom end.

Proposition 1. Suppose $\Sigma$ is a compact and embedded $H$-surface in $N=M_{[a, \infty)}$ with boundary in $M_{a}^{2}$. If the Gauss curvature of $M^{2}$ satisfies $K_{M} \geq 2 \tau(\tau \leq 0)$ and $H^{2}>|\tau|$, then

$$
\Sigma \subset M_{[a, a+2 c]},
$$

where $c=\frac{H}{H^{2}-|\tau|}$. In particular, if the sectional curvature of $M^{2}$ is nonnegative and $H \neq 0$, then

$$
\Sigma \subset M_{\left[a, a+\frac{2}{H}\right]} .
$$

Our proof of Proposition 1 (and of Proposition 2 below) uses a height estimate for $H$-surfaces that are graphs in $M^{2} \times \mathbf{R}$ with zero boundary values over compact domains in $M_{0}^{2}$. Let $h: \Sigma \rightarrow \mathbf{R}$ be the restriction of the projection $t: M^{2} \times \mathbf{R} \rightarrow \mathbf{R}$, to $\Sigma$. We refer to $h$ as the height function.

Lemma 1. Suppose that the Gauss curvature of $M^{2}$ satisfies $K_{M} \geq 2 \tau(\tau \leq 0)$. Let $W$ be an $H$-surface with $H^{2} \geq|\tau|$, which is a graph over a compact region in $M_{0}^{2}$, with zero boundary values. Then

$$
h \leq \frac{H}{H^{2}-|\tau|}
$$


on $W$. In particular, if the Gauss curvature of $M^{2}$ is nonnegative and $H \neq 0$, then

$$
h \leq \frac{1}{H}
$$

We will provide the proof of Lemma 1 at the end of the section.

Proof of Proposition 1. Observe that translation by $t_{0}$ (i.e. $M_{t}^{2} \rightarrow M_{t+t_{0}}^{2}$ ) is an isometry of $N=M^{2} \times \mathbf{R}$. Therefore, without loss of generality, we may assume that there exists $b>0$ for which $\Sigma \subset M_{[0, b]}$, with $\partial \Sigma \subset M_{0}^{2}$ and $\Sigma \cap M_{b}^{2} \neq \emptyset$ and $\Sigma \cap M_{s}^{2}=\emptyset$ for $s>b$. To prove (1), we must show that $\frac{b}{2}<c$.

For $|b-t|$ small, $\Sigma \cap M_{[t, b]}$ is a vertical graph over a compact domain in $M_{0}^{2}$. By the Alexandrov reflection technique, the part of $\Sigma$ in $M_{\left[\frac{b}{2}, b\right]}$ is also a graph over a compact domain in $M_{0}^{2}$. To see this, first observe that since reflection in $M_{t}$ cannot be a symmetry of $\Sigma$ for $t>\frac{b}{2}$, one can do Alexandrov reflection through all the $M_{\tau}^{2}$, as $\tau$ decreases from $b$ to $\frac{b}{2}$, and no accident occurs. That is, the part of $\Sigma$ above each $M_{\tau}^{2}$ is a vertical graph for $\frac{b}{2} \leq \tau \leq b$. By Lemma 1, $\frac{b}{2}<c$, which gives (11). The second statement, (2), follows immediately from the first.

We will say that a surface in $M^{2} \times \mathbf{R}$ lies in a halfspace if it is contained in a region of $M^{2} \times \mathbf{R}$ of the form

$$
M_{[a, \infty)}=\left\{(p, t) \in M^{2} \times \mathbf{R} \mid t \geq a\right\} \quad \text { or } \quad M_{(-\infty, a]}=\left\{(p, t) \in M^{2} \times \mathbf{R} \mid t \leq a\right\} .
$$

Proposition 2. Suppose $M^{2}$ is a compact surface without boundary whose Gauss curvature is bounded below by $2 \tau$, for some real number $\tau$, and suppose $\Sigma$ is a noncompact properly embedded $H$ surface in $N=M^{2} \times \mathbf{R}$. If $\tau<0$, assume that $H^{2} \geq|\tau|$. Then $\Sigma$ cannot lie in a halfspace. In particular, $\Sigma$ must have at least one "top" and one "bottom" end.

Proof. We first prove that $H \neq 0$ if $\Sigma$ lies in a halfspace. This is because we can find an $M_{t}^{2}$ tangent to $\Sigma$ at a point, with $M^{2}$ lying on one side of $\Sigma$. By the maximum principle we would have $M_{t}^{2}=\Sigma$, a contradiction since $M^{2}$ is compact and $\Sigma$ is not. This means that we satisfy the conditions of Lemma 1 even when $M^{2}$ has nonnegative curvature.

Observe that translation by $t_{0}$ (i.e., $M_{t}^{2} \rightarrow M_{t+t_{0}}^{2}$ ) and reflection $R_{t_{0}}$ in $M_{t_{0}}^{2}$ (i.e., $M_{t}^{2} \rightarrow M_{2 t_{0}-t}^{2}$ ) are isometries of $N=M^{2} \times \mathbf{R}$. Therefore, without loss of generality, we may assume that $\Sigma$ lies in the halfspace $M_{[0, \infty)}$ and has a nonempty intersection with $M_{0}$.

Since $\Sigma_{a}:=\{(x, t) \in \Sigma \mid t \leq \alpha\}$ is compact, we may do Alexandrov-reflection coming up from $M_{0}^{2}$. For any value of $a, R_{a}\left(\Sigma_{a}\right)$ is compact. Since $\Sigma$ is not compact, $R_{a}$ is not a symmetry of $\Sigma$. This implies that for every $a>0$, the compact surface $\Sigma_{a}$ must be a vertical graph over a domain in $M_{a}$. However, the hypotheses of this proposition on the curvature of $M^{2}$ and the lower bounds for $H$ allow us to use Lemma 1 for such a vertical graph, providing a height estimate that is violated for $a>0$ sufficiently large. This contradiction establishes the first statement of the proposition. The second statement is an immediate consequence of the first.

Proof of Lemma 1. We begin with some necessary general observations. For any immersion $X: \Sigma \rightarrow N$ of one Riemannian manifold into another, the Laplacian of 
$X$ is the divergence of the vector-valued one form $D X$

$$
\Delta X:=\sum_{i=1}^{m}\left[\nabla_{D X\left(e_{i}\right)} D X\left(e_{i}\right)-D X\left(\hat{\nabla}_{e_{i}} e_{i}\right)\right]
$$

where $\nabla$ and $\hat{\nabla}$ are the Riemannian connections on $N$ and $\Sigma$, respectively, and the $\left\{e_{i}\right\}$ are an orthonormal framing of $T \Sigma$. If $X$ is an isometric immersion, then $D X\left(\hat{\nabla}_{e_{i}} e_{i}\right)=\left[\nabla_{D X\left(e_{i}\right)} D X\left(e_{i}\right)\right]^{t}$, where [ $]^{t}$ is the projection of $T N_{\mid X(\Sigma)}$ onto $X_{*}(T \Sigma)$. Then

$$
\Delta X=\sum_{i=1}^{m}\left[\nabla_{D X\left(e_{i}\right)} D X\left(e_{i}\right)\right]^{n}=\sum_{i=1}^{m} B\left(e_{i}, e_{i}\right),
$$

where [ ] ${ }^{n}$ is projection onto the normal bundle of $X(\Sigma) \subset N$ and $B$ is the second fundamental form of $X$. [13] Therefore, the Laplacian of $X$ is the trace of the second fundamental form of $\Sigma$,

$$
\Delta X=m \vec{H}
$$

where $m$ is the dimension of $\Sigma$ and $\vec{H}$ is the mean curvature vector field of the isometric immersion $X$.

In our context, $N=M^{2} \times \mathbf{R}$. Let $\vec{n}$ denote the unit normal to $\Sigma$ and define

$$
H:=\langle\vec{H}, \vec{n}\rangle \text { and } n:=\left\langle\vec{n}, \frac{\partial}{\partial t}\right\rangle
$$

We have

$$
\Delta X=2 H \vec{n} .
$$

A simple calculation using either the fact that the height function, $h$, is the projection onto a one-dimensional subspace in a Riemannian splitting, or that $\frac{\partial}{\partial t}$ is a Killing field, gives

$$
\Delta h=2 H n \text {. }
$$

We will also have need of an equation for the Laplacian of $n=\left\langle\vec{n}, \frac{\partial}{\partial t}\right\rangle$ :

$$
\Delta n=-\left(|A|^{2}+\operatorname{Ric}(\vec{n})\right) n,
$$

where $A$ is the shape operator of $\Sigma$. (Recall that $A: T \Sigma \rightarrow T \Sigma$ with $A(U)=\nabla_{U} \vec{n}$.) Formula (6) can be derived as in 22, or [18 by looking at the second variation of area of an $H$-surface in the direction of a normal component of a Killing field (in this case $\frac{\partial}{\partial t}$ ). Alternatively, one can derive this equation, directly using the fact that $\frac{\partial}{\partial t}$ is a Killing field. From general considerations,

$$
\Delta n=\Delta\left\langle\vec{n}, \frac{\partial}{\partial t}\right\rangle=\left\langle\Delta \vec{n}, \frac{\partial}{\partial t}\right\rangle+2\left\langle\nabla \vec{n}, \nabla \frac{\partial}{\partial t}\right\rangle+\left\langle\vec{n}, \Delta \frac{\partial}{\partial t}\right\rangle .
$$

Because $\frac{\partial}{\partial t}$ is Killing, $\left\langle\nabla_{U} \frac{\partial}{\partial t}, V\right\rangle$ is skew symmetric, which is enough to show that the middle term on the right-hand side of the above expression vanishes. It also allows expression of the last summand in terms if $\operatorname{Ric}\left(\vec{n}, \frac{\partial}{\partial t}\right)$. Simplification leads to (6).

Now we restrict our attention to an $H$-surface $W$ that satisfies the assumptions of the lemma. Define

$$
\phi=c h+n,
$$


where $c$ is a real-valued positive constant. Since $W$ is a graph we may choose a "downward-pointing" unit normal vector field $\vec{n}$ so that

$$
n:=\left\langle\vec{n}, \frac{\partial}{\partial t}\right\rangle \leq 0
$$

on $W$. Since $W$ is assumed to be a graph with zero boundary values, it follows that

$$
\phi=n \leq 0
$$

on $\partial W$. If we can find a value of $c$ for which $\Delta \phi \geq 0$ on $W$, it will follow from the maximum principle that $\phi=c h+n \leq 0$ on $W$; hence

$$
h \leq-n / c \leq 1 / c .
$$

We will now find such a value of $c$. From (5) and (6), we get

$$
\Delta \phi=\left(2 c H-|A|^{2}-\operatorname{Ric}(\vec{n})\right) n .
$$

Since $n \leq 0$, we can assert that $\Delta \phi \geq 0$ if and only if

$$
|A|^{2}+\operatorname{Ric}(\vec{n}) \geq 2 c H \text {. }
$$

Using the simple estimate

$$
\begin{aligned}
|A|^{2} & =\operatorname{tr}^{2}(A)-2 \operatorname{det}(A) \\
& =2\left(\frac{\operatorname{tr}(A)}{2}\right)^{2}+\left(\frac{1}{2} \operatorname{tr}^{2}(A)-2 \operatorname{det}(A)\right) \\
& =2 H^{2}+\frac{1}{2}\left(a_{11}-a_{22}\right)^{2}+2 a_{12}^{2} \\
& \geq 2 H^{2},
\end{aligned}
$$

it suffices to choose $c$ so that

$$
H^{2}+\frac{1}{2} \operatorname{Ric}(\vec{n}) \geq c H
$$

Since $\operatorname{Ric}(\vec{n})=|\operatorname{Pr}(\vec{n})|^{2} K_{M}$, where $\operatorname{Pr}\left(\right.$ ) is the projection from $T N$ onto $T M^{2}$, and $|n|=1$, it follows from our assumption that $K_{M} \geq 2 \tau, \tau \leq 0$, and that $\operatorname{Ric}(\vec{n}) \geq 2 \tau$. Therefore we can satisfy the condition on $c$ above with

$$
\frac{H^{2}-|\tau|}{H} \geq c
$$

The left-hand side of (10) is positive by assumption and so choosing $c$ to equal the left-hand side is sufficient to force $\Delta \phi \geq 0$. The height estimate (3) follows from (8).

If $M^{2}$ has nonnegative sectional curvature, then $\operatorname{Ric}(N) \geq 0$, the requirement (10) holds for $c=H$, and (8) gives the height estimate (4).

Remark 1. The height estimate in Lemma 1 in the case that $M^{2} \times \mathbf{R}=H^{2} \times \mathbf{R}$ works for $H>\frac{1}{\sqrt{2}}$. However the rotational $H$-surfaces in $H^{2} \times \mathbf{R}$ are compact for $H>1 / 2$, and noncompact for $H \leq \frac{1}{2}$, [17. So one suspects our height estimates are not sharp. 


\section{First VARIATIONS OF AREA AND VOLUme}

Let $U$ be bounded domain in a Riemannian three manifold $N$, whose boundary, $\partial U$, consists of a smooth connected surface, $\Sigma$, and the union $Q$ of finitely many smooth, compact and connected surfaces. The closed surface $\partial U$ is piecewisesmooth and smooth except perhaps on $\partial \Sigma=\partial Q$. Let

$$
\begin{aligned}
\vec{n} & =\text { the outward-pointing unit normal vector field on } \partial U=\Sigma \cup Q ; \\
\vec{n}_{\Sigma} & =\text { the restriction of } \vec{n} \text { to } \Sigma ; \\
\vec{n}_{Q} & =\text { the restriction of } \vec{n} \text { to } Q .
\end{aligned}
$$

Suppose $Y$ is a vector field defined on a region of $N$ that contains $U$. The first-variation of the volume, $|U|$, of $U$ is given by

$$
\begin{aligned}
\delta_{Y}|U|=\int_{U} \operatorname{Div} Y & =\int_{\partial U} Y \cdot \vec{n} \\
& =\int_{\Sigma} Y \cdot \vec{n}_{\Sigma}+\int_{Q} Y \cdot \vec{n}_{Q}
\end{aligned}
$$

where $\operatorname{Div}=\operatorname{Div}_{N}$ is the divergence operator on $N$. To write down $\delta_{Y}(|\Sigma|)$, the first variation of area of $\Sigma$ under $Y$, we introduce the following notation:

$$
\begin{aligned}
\nu & =\text { the outward-pointing unit conormal to } \Sigma \text { along } \partial \Sigma ; \\
Y^{t} & =\text { the tangential projection of } Y \text { onto } T \Sigma ; \\
Y^{n} & =Y-Y^{t}, \text { the projection of } Y \text { onto the normal bundle of } \Sigma \text { in } N .
\end{aligned}
$$

We may then write

$$
\begin{aligned}
\delta_{Y}(|\Sigma|) & =\int_{\Sigma} \operatorname{div} Y \\
& =\int_{\Sigma} \operatorname{div} Y^{t}+\int_{\Sigma} \operatorname{div} Y^{n} \\
& =\int_{\partial \Sigma} Y \cdot \nu-\int_{\Sigma} Y \cdot \vec{H}_{\Sigma}
\end{aligned}
$$

where $d i v=d i v_{\Sigma}$, is the divergence operator on $\Sigma$.

If $\Sigma$ is an $H$-surface, $H:=\langle\vec{H}, \vec{n}\rangle$ is a constant and therefore

$$
\delta_{Y}(|\Sigma|)=\int_{\partial \Sigma} Y \cdot \nu-H \int_{\Sigma} Y \cdot \vec{n} .
$$

Putting (11) and(12) together, we have for $H$-surfaces,

$$
\delta_{Y}(|\Sigma|+H|U|)=\int_{\partial \Sigma} Y \cdot \nu+H \int_{Q} Y \cdot \vec{n}_{Q} .
$$

The following propostion is well known and is immediate from (13).

Proposition 3. If $Y$ is a Killing vector field on $N$, and $\Sigma$ is an $H$-surface, then

$$
\int_{\partial \Sigma} Y \cdot \nu+H \int_{Q} Y \cdot \vec{n}_{Q}=0,
$$

where $Q, \nu, H$ and $\vec{n}_{Q}$ are as defined above. 
Suppose $\Sigma$ is a properly embedded $H$-surface in $N=M^{2} \times \mathbf{R} /$, and assume that $\Sigma$ bounds a region $U \subset N$. Define:

$$
\begin{aligned}
M_{t} & =M^{2} \times\{t\} ; \\
\Sigma_{t} & =\Sigma \cap M_{t} ; \\
\Sigma_{[a, b]} & =\Sigma \cap\left(M^{2} \times[a, b]\right)=\bigcup_{a \leq s \leq b} \Sigma_{s} ; \\
U_{a}^{b} & =U \cap\left(M^{2} \times[a, b]\right) ; \\
Q_{t} & =U \cap M_{t}
\end{aligned}
$$

Clearly,

$$
\partial U_{a}^{b}=\Sigma_{[a, b]} \cup Q_{a} \cup Q_{b}, \quad \partial \Sigma=\Sigma_{a} \cup \Sigma_{b}, \quad \vec{n}_{Q_{b}}=+\frac{\partial}{\partial t}, \quad \vec{n}_{Q_{a}}=-\frac{\partial}{\partial t} .
$$

We now apply Proposition 3 to the vector field $\frac{\partial}{\partial t}$. Since vertical translation $M_{t} \rightarrow M_{t+t_{0}}$ is an isometry, $\frac{\partial}{\partial t}$ is a Killing field and we have from (14)

$$
\begin{aligned}
0 & =\int_{\Sigma_{0} \cup \Sigma_{t}} \frac{\partial}{\partial t} \cdot \nu+H \int_{Q} \frac{\partial}{\partial t} \cdot \vec{n}_{Q} \\
& =\int_{\Sigma_{0}} \frac{\partial}{\partial t} \cdot \nu+\int_{\Sigma_{t}} \frac{\partial}{\partial t} \cdot \nu+H\left(\left|Q_{t}\right|-\left|Q_{0}\right|\right) .
\end{aligned}
$$

We will state an important consequence of this computation as a proposition for later use.

Proposition 4. Suppose $\Sigma$ is a properly embedded $H$-surface in $N=M^{2} \times \mathbf{R}$, $M^{2}$ compact. Then the vertical flux across $\Sigma_{t}$,

$$
\int_{\Sigma_{t}} \frac{\partial}{\partial t} \cdot \nu
$$

varies within a bounded range

The proposition follows immediately from (17) and the assumption that $M^{2}$ is compact: since $Q_{t} \subset M_{t}$, the volume $\left|Q_{t}\right|$ is bounded independent of $t$.

Remark 2. If $\Sigma$ is a minimal surface $(H=0)$, then it follows from (17) that the $\int_{\Sigma_{t}} \frac{\partial}{\partial t} \cdot \nu$, the vertical flux across $\Sigma_{t}$, is a constant, independent of $t$.

\section{Linear AREA GROWTH}

In this section, we prove that a properly embedded $H$-surface in $N=S^{2} \times \mathbf{R}$ will have linear area growth provided it has one additional property. For any choice of antipodal points on $S^{2}$, consider the rotations of $S^{2}$ that fix these two points. These extend to rotations of $N$ whose common fixed point set consists of the two vertical geodesics through these points. Choose a geodesic arc $\gamma$ joining these antipodal points and let $\mathcal{P}$ be the union of $\gamma \times \mathbf{R}$ and any tubular neigborhoods of the two vertical geodesics.

Theorem 1. Let $\Sigma$ be a properly embedded $H$-surface in $N=S^{2} \times \mathbf{R}$. If $\Sigma$ is disjoint from $\mathcal{P}$, then $\Sigma$ has linear area growth.

A generalization of this theorem is given in Section 4.1. 
Remark 3. In the complement of $\mathcal{P}$ we may define $\theta$, the angle measured from $\gamma$. The gradient of $\theta$ is a Killing field whose length is bounded above by 1 , and below by a positive constant that depends on the radius of the tubular neigborhoods chosen in the definition of $\mathcal{P}$.

Proof. Given $a>0$, we will show that

$$
\left|\Sigma_{[-a, a]}\right| \leq c a
$$

for some constant $c$ that does not depend on $a$.

Note that $(x, t) \rightarrow(x,-t)$ is an isometry of $N$, and therefore

$$
\left|\Sigma_{[-a, a]}\right|=\left|-\Sigma_{[-a, a]}\right|,
$$

where $-\Sigma=\{(x,-t) \mid(x, t) \in \Sigma\}$. Hence, by working with $-\Sigma$ if necessary, we may assume, without loss of generality, that

$$
\left|\Sigma_{[-a, 0]}\right| \leq\left|\Sigma_{[0, a]}\right|
$$

Since $(x, t) \rightarrow(x, t+a)$ is an isometry of $N$, the inequality (18) will follow if we establish

$$
\left|\Sigma_{[0,2 a]}\right| \leq c a, \text { assuming }\left|\Sigma_{[0, a]}\right| \leq\left|\Sigma_{[a, 2 a]}\right| .
$$

We will now prove (19).

On $S^{2}-\gamma$, we may define polar coordinates, $(r, \theta)$, with $\pi<\theta<3 \pi$. The function $\theta$ is well defined on $N-\mathcal{P}$, a region that contains $\Sigma$ by assumption.

Let $\frac{\partial}{\partial \theta}$ be the rotational Killing field for the axis defined by the end-points of $\gamma$, and let $\frac{\partial}{\partial t}$ be the Killing field defined by vertical translation. Clearly, $\left|\frac{\partial}{\partial t}\right|=1$ and, as noted in Remark [3, $\left|\frac{\partial}{\partial \theta}\right|$ is bounded above and also bounded away from zero. Define the vector fields

$$
X=t \frac{\partial}{\partial t}, \quad Y=\theta \frac{\partial}{\partial \theta}, \quad Z=t Y .
$$

A direct calculation using

$$
\operatorname{div} W=\operatorname{Div} W-\left\langle\nabla_{n} W, n\right\rangle, \quad \operatorname{Divf} W=\langle\nabla f, W\rangle+f \operatorname{Div} W,
$$

and the fact that for a Killing field $V,\left\langle\nabla_{e} V, e\right\rangle=0$ and therefore

$$
\operatorname{Div} V=\operatorname{div} V=0 \text {, and }\left\langle\nabla_{n} V, n\right\rangle=0,
$$

gives

$$
\begin{aligned}
& \operatorname{Div} X=1, \quad \operatorname{div} X=1-n_{t}^{2}, \\
& \operatorname{Div} Y=\left|\frac{\partial}{\partial \theta}\right|^{2}, \quad \operatorname{div} Y=\left|\frac{\partial}{\partial \theta}\right|^{2}-n_{\theta}^{2}, \\
& \operatorname{Div} Z=t\left|\frac{\partial}{\partial \theta}\right|^{2}, \quad \operatorname{div} Z=t\left(\left|\frac{\partial}{\partial \theta}\right|^{2}-n_{\theta}^{2}\right)-\theta n_{t} n_{\theta},
\end{aligned}
$$

where $n_{t}=\overrightarrow{n_{\Sigma}} \cdot \frac{\partial}{\partial t}$ and $n_{\theta}=\overrightarrow{n_{\Sigma}} \cdot \frac{\partial}{\partial \theta}$. Using $X$ as the vector field in the first variation formula (13) on $\Sigma_{[0,2 a]}$, we have

$$
\begin{aligned}
\int_{\Sigma_{[0,2 a]}\left(1-n_{t}^{2}\right)+H\left|U_{0}^{2 a}\right|} & =\int_{\Sigma_{0} \cup \Sigma_{2 a}} t \frac{\partial}{\partial t} \cdot \nu+H \int_{Q_{0} \cup Q_{2 a}} t \frac{\partial}{\partial t} \cdot \overrightarrow{n_{Q}} \\
& =2 a\left[\int_{\Sigma_{2 a}} \frac{\partial}{\partial t} \cdot \nu+H \int_{Q_{2 a}} \frac{\partial}{\partial t} \cdot \overrightarrow{n_{Q}}\right] \\
& =2 a\left[\int_{\Sigma_{2 a}} \frac{\partial}{\partial t} \cdot \nu+H\left|Q_{2 a}\right|\right] .
\end{aligned}
$$




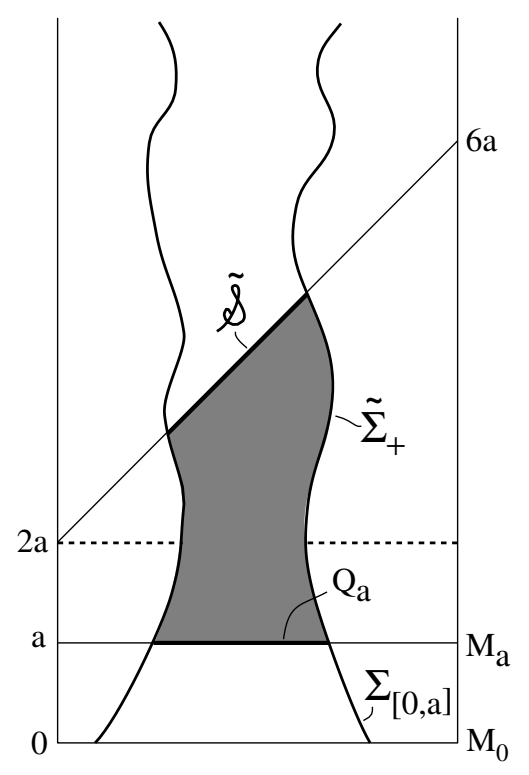

FIGURE 1. $\tilde{\Sigma}$.

By Proposition 3, equation (14), the bracketed term on the right-hand side is a constant. Moreover, since $S^{2}$ has finite area, $\left|U_{0}^{2 a}\right|$ grows at most linearly in $a$. Hence

$$
\int_{\Sigma_{[0,2 a]}} 1-n_{t}^{2} \leq c_{1} a
$$

where $c_{1}$ does not depend on $a$.

We now use (13) with the variation vector field $Z$. This time we will cut off $\Sigma$ below by $S_{0}^{2}=S^{2} \times\{0\}$, and above by a surface, $\mathcal{S}$, defined by

$$
t \theta=6 \pi a .
$$

Note that when $t=2 a$, we have $\theta=3 \pi$, and when $t=6 a$, we have $\theta=\pi$. (See Figure 1.) We will denote the parts of $\Sigma$ and $U$ between $S_{0}^{2}$ and $\mathcal{S}$ by $\tilde{\Sigma}$ and $\tilde{U}$, respectively, and define $\tilde{\mathcal{S}}=\mathcal{S} \cap U$ and $\Sigma_{\mathcal{S}}=\Sigma \cap \mathcal{S}$. (See Figure 1, again.) We get from (13)

$$
\begin{aligned}
\int_{\tilde{\Sigma}}( & \left.t\left(\left|\frac{\partial}{\partial \theta}\right|^{2}-n_{\theta}^{2}\right)-\theta n_{t} n_{\theta}\right)-H \int_{\tilde{U}}\left|\frac{\partial}{\partial \theta}\right|^{2} t \\
\quad= & \int_{\Sigma_{0} \cup \Sigma_{\mathcal{S}}} Z \cdot \nu+H \int_{Q_{0} \cup \tilde{\mathcal{S}}} Z \cdot \overrightarrow{n_{Q}}=6 \pi a\left[\int_{\Sigma_{\mathcal{S}}} \frac{\partial}{\partial \theta} \cdot \nu+H \int_{\tilde{\mathcal{S}}} \frac{\partial}{\partial \theta} \cdot \overrightarrow{n_{Q}}\right] .
\end{aligned}
$$

Since $\frac{\partial}{\partial \theta}$ is a Killing field, it follows from Proposition 3, equation (14), that the bracketed term on the right is a constant that does not depend on $a$. Hence

$$
\int_{\tilde{\Sigma}} t\left(\left|\frac{\partial}{\partial \theta}\right|^{2}-n_{\theta}^{2}\right)=c_{2} a+H \int_{\tilde{U}} t\left|\frac{\partial}{\partial \theta}\right|^{2}+\int_{\tilde{\Sigma}} \theta n_{t} n_{\theta} .
$$

Now observe that $\pi<\theta<3 \pi$ on $\tilde{\Sigma}$; the vector fields $\vec{n}$ and $\frac{\partial}{\partial t}$ both have length 1 . Moreover, the Killing field $\frac{\partial}{\partial \theta}$ has length bounded above and is also bounded away 
from zero on $\tilde{\Sigma}$ :

$$
0<c_{*} \leq\left|\frac{\partial}{\partial \theta}\right| \leq c^{*}
$$

Therefore

$$
\left|\theta n_{t} n_{\theta}\right| \leq 3 \pi n_{t}=3 \pi\left\langle n, \frac{\partial}{\partial \theta}\right\rangle \leq 3 \pi c^{*}:=c_{4} .
$$

Also note that $0 \leq t \leq 6 a$ on $\tilde{U}$. Using these estimates we have

$$
\begin{aligned}
\int_{\tilde{\Sigma}} t\left(\left|\frac{\partial}{\partial \theta}\right|^{2}-n_{\theta}^{2}\right) & \leq c_{2} a+c_{3} a^{2}+\int_{\tilde{\Sigma}} \theta n_{t} n_{\theta} \\
& \leq c_{2} a+c_{3} a^{2}+c_{4}|\tilde{\Sigma}| .
\end{aligned}
$$

Here we have used the fact that $S^{2}$ has finite area and therefore the volume of $|\tilde{U}|$ is linear in $a$.

Let $\tilde{\Sigma}_{+}=\tilde{\Sigma} \cap M_{[a, 6 a]}$. We are assuming that $\left|\Sigma_{[0, a]}\right| \leq\left|\Sigma_{[a, 2 a]}\right|$, and since $\Sigma_{[a, 2 a]} \subset \tilde{\Sigma}_{+}$

$$
|\tilde{\Sigma}|=\left|\Sigma_{[0, a]}\right|+\left|\tilde{\Sigma}_{+}\right| \leq 2\left|\tilde{\Sigma}_{+}\right| .
$$

From (24), (23) and (22), we get

$$
\begin{aligned}
a c_{*}^{2} \int_{\tilde{\Sigma}_{+}}\left(1-\left(\frac{n_{\theta}}{\left|\frac{\partial}{\partial \theta}\right|}\right)^{2}\right) & \leq \int_{\tilde{\Sigma}_{+}} t\left|\frac{\partial}{\partial \theta}\right|^{2}\left(1-n_{\theta}^{2}\right) \\
& \leq \int_{\tilde{\Sigma}} t\left|\frac{\partial}{\partial \theta}\right|^{2}\left(1-n_{\theta}^{2}\right) \leq c_{2} a+c_{3} a^{2}+2 c_{4}\left|\tilde{\Sigma}_{+}\right| .
\end{aligned}
$$

Dividing by $a c_{*}^{2}$ gives

$$
\int_{\tilde{\Sigma}_{+}}\left(1-\left(\frac{n_{\theta}}{\left|\frac{\partial}{\partial \theta}\right|}\right)^{2}\right) \leq \frac{1}{c_{*}^{2}}\left(c_{2}+c_{3} a+\frac{2 c_{4}}{a}\left|\tilde{\Sigma}_{+}\right|\right) .
$$

In estimate (21), we saw that $\int_{\Sigma_{[0,2 \hat{a}]}} 1-n_{t}^{2} \leq c_{1} \hat{a}$, where $c_{1}$ does not depend on $\hat{a}$. Using $\hat{a}=3 a$ and observing that $\tilde{\Sigma}_{+} \subset \Sigma_{[0,6 a]}$, we get (using the fact that $\left.n_{t}^{2}+\left(\frac{n_{\theta}}{\left|\frac{\partial}{\partial \theta}\right|}\right)^{2} \leq 1\right)$

$\left|\tilde{\Sigma}_{+}\right| \leq \int_{\tilde{\Sigma}_{+}}\left(2-\left(n_{t}^{2}+\left(\frac{n_{\theta}}{\left|\frac{\partial}{\partial \theta}\right|}\right)^{2}\right) \leq\left(\frac{1}{c_{*}^{2}}\left(c_{2}+c_{3} a\right)+3 c_{1} a\right)+\frac{2 c_{4}}{a c_{*}^{2}}\left|\tilde{\Sigma}_{+}\right|=c_{5}+c_{6} a+\frac{2 c_{4}}{a c_{*}^{2}}\left|\tilde{\Sigma}_{+}\right|\right.$.

If $a>\frac{4 c_{4}}{c_{*}^{2}}$, then

$$
\left|\tilde{\Sigma}_{+}\right| \leq 2\left(c_{5}+c_{6} a\right) .
$$

This estimate together with (24) produces

$$
|\tilde{\Sigma}| \leq 4\left(c_{5}+c_{6} a\right)
$$

from which (19) follows because $\Sigma_{[0,2 a]} \subset \tilde{\Sigma}$. This completes the proof of the theorem. 
4.1. Linear area growth in $M^{2} \times \mathbf{R}$ when $M^{2}$ has a pole. The proof of Theorem 1 will give linear area growth in more general situations. Suppose $M^{2}$ has a pole, $\mathbf{p}$, a fixed point for a one-parameter group of rotational isometries. Assume there is a region $\mathcal{R} \subset N$, inside of which the angle of rotation is well defined and the gradient of that angle (a Killing field) has length bounded above and also away from zero. Let $\Sigma \subset \mathcal{R}$ be a properly embedded $H$-surface, and assume that $\Sigma$ bounds a region $U \subset \mathcal{R}$ with the property that the area of $U_{t}=U \cap M_{t}^{2}$ is uniformly bounded. Then the proof of Theorem 1 is directly generalizable to a proof of the linear area growth of $\Sigma$. In particular

Theorem 2. Suppose $M^{2}$ is a complete surface with a pole $\mathbf{p}$. The Killing field $\frac{\partial}{\partial \theta}$ is defined in a punctured neighborhood, say $D$, of $\mathbf{p}$, and the angle $\theta$ is defined in the complement in $D$ of a geodesic, say $\gamma$, starting at $\mathbf{p}$ and running to the cut locus $C_{\mathbf{p}}$ of $\mathbf{p}$. Let $\mathcal{R}=(D \backslash \gamma) \times \mathbf{R}$, and suppose that $\Sigma=\partial U, U \subset \mathcal{R}$, is a properly embeddded $H$-surface. Suppose further that $\left|U_{t}\right|=\left|U \cap M_{t}^{2}\right|$ is bounded independent of $t$ and that the Killing field $\frac{\partial}{\partial \theta}$ associated with the pole $\mathbf{p}$ satisfies the boundedness condition (22). Then $\Sigma$ has linear area growth.

This theorem has an immediate corollary when $M^{2}=H^{2}$.

Corollary 1. i) Suppose $\gamma \subset H^{2}$ is a geodesic ray beginning at $\mathbf{p}$. Let $\Sigma=$ $\partial U \subset \mathcal{R}=\left(H^{2} \backslash \gamma\right) \times \mathbf{R}$ be a properly embedded $H$-surface with the property that $\left|U_{t}\right|$ is bounded independent of $t$. Suppose further that the Killing field $\frac{\partial}{\partial \theta}$ associated with the pole $\mathbf{p}$ satisfies the boundedness condition of (22). Then $\Sigma$ has linear area growth.

ii) Suppose $\alpha: \mathbf{R} \rightarrow H^{2}$ is either a constant map or a constant-speed $(=b)$ parametrization of a complete geodesic. Let $\beta(t)=(\alpha(t), c t), c \neq 0, b^{2}+$ $c^{2}=1$, be a parametrization of a complete unit-speed geodesic $\beta \subset H^{2} \times \mathbf{R}$. Suppose $\Sigma \subset H^{2} \times \mathbf{R}$ is a properly embedded $H$-surface which is cylindrically bounded in the sense that it stays a bounded distance from $\beta$. Then $\Sigma$ has linear area growth.

Proof. The first statement follows directly from Theorem 2 and the fact that any point $\mathbf{p} \in H^{2}$ is a pole whose cut locus is empty.

To prove the second statement, we work in the disk model of $H^{2}$. We first observe that without loss of generality, we may assume that either $\alpha(t)$ is indentically equal to zero, or is a constant-speed parametrization (in $H^{2}$ ) of the real axis. Furthermore, we may assume that $\Sigma$ is within distance one of $\beta$.

If $\beta(t)=(0, c t)$, choose $\mathbf{p}$ to be a point on the imaginary axis at distance $d>3$ from the origin, and let $\gamma$ be the geodesic ray beginning at $\mathbf{p}$ and diverging along the imaginary axis. Let $\mathcal{R}=B_{2}(0) \times \mathbf{R} \subset H^{2} \times \mathbf{R}$. All the conditions of statement (i) are now satisfied, so $\Sigma$ has linear area growth.

If $\alpha$ is the real axis and the cylindrical radius is one, we again choose $\gamma$ as before. Let $\mathcal{B}=B \times \mathbb{R}$, where $B$ is the geodesic disc in $\mathbb{H}^{2}$ centered at $\beta(0)=O$, and of radius 3. Clearly $\Sigma_{[0, c]} \subset \mathcal{B}$. Our previous calculations in Theorem 1 show that the area of $\Sigma_{[0, c]}$ is bounded by $k c$, where the constant $k$ depends on the bounds for $\left|\partial_{\theta}\right|$ in $\mathcal{B}$. Observe that the same estimate holds for the area of $\Sigma_{[c, 2 c]}$, since there is an isometry $\phi$ of $\mathbb{H}^{2} \times \mathbb{R}$ to itself, leaving $\beta$ invariant, taking $\beta(1)$ to $\beta(0) ; \phi^{-1}$ is the composition of the (hyperbolic) translation of $\mathbb{H}^{2}$, taking $O$ to $\alpha(1)$, with the vertical translation taking $\alpha(1)$ to $\beta(1)$. This map takes $\beta^{\prime}(0)$ to $\beta^{\prime}(1)$, and hence 
leaves $\beta$ invariant. Then $\phi\left(\Sigma_{[c, 2 c]}\right) \subset \mathcal{B}$, so its area is also at most $k c$. Continuing this way, we conclude $\Sigma$ has linear (vertical) area growth.

\section{Curvature Bounds}

We will be concerned in this section with properly embedded $H$-surfaces, $\Sigma \subset$ $N=M^{2} \times R$, of finite topology with the additional property that the restriction of the height function on $M^{2} \times \mathbf{R}$ to $\Sigma$ is proper. If $M^{2}$ is compact, every such $\Sigma$ has this property. Note that an annular end of such an $H$-surface must be either a top end or a bottom end.

We will assume that $M^{2}$ is complete, that $M^{2}$ has bounded Gauss curvature, and that the injectivity radius of $M^{2}$ is bounded away from zero. Again, all these properties hold if $M^{2}$ is compact.

Theorem 3. Let $\Sigma$ and $M^{2}$ satisfy the above conditions. If $\Sigma$ has linear area growth, then $|A|$ is bounded on $\Sigma$.

Corollary 2. Suppose $\Sigma$ is a properly embedded $H$-surface with finite topology in $S^{2} \times \mathbf{R}$, with the additional property that $\Sigma \subset D \times R \subset S^{2} \times \mathbf{R}$, where $D$ is a geodesic disk of radius strictly less than $\pi$ (or, more generally satisfies the conditions of Theorem 1). Then $|A|$ is bounded on $\Sigma$.

The corollary follows immediately from Theorem 3 since $S^{2}$ is compact and Theorem 1 gives linear area growth. Similar results for $H$-surfaces $\Sigma \subset H^{2} \times R$ on which the height function is proper follow from Theorem 3 and Corollary 1 .

Theorem 3 will be proved after we present two preliminary results.

We begin by observing as we $\operatorname{did}$ in (10), that $|A|^{2}=\operatorname{tr}^{2}(A)-2 \operatorname{det}(A)=4 H^{2}-$ $2 \operatorname{det}(A)$ - a local formula not requiring any of our hypotheses - and that the Gauss curvature $K(p)$ of $\Sigma$ at a point $p \in \Sigma$ satisfies $\operatorname{det}\left(A_{p}\right)=K(p)-K_{N}(p)$, where $K_{N}(p)$ is the sectional curvature of $N$ on the plane $T_{p} \Sigma$. Therefore

$$
|A|^{2}=4 H^{2}+2 K_{N}-2 K \text {. }
$$

The geometric quantity $4 H^{2}+2 K_{N}$ is bounded on $\Sigma$ if $M^{2}$ has bounded curvature. Therefore

Lemma 2. Let $\Sigma=\partial U, U \subset N=M^{2} \times \mathbf{R}$, be a properly embedded $H$-surface. Assume $\Sigma$ has linear area growth and that $M^{2}$ has bounded curvature. Then

$$
\int_{\Sigma} 4 H^{2}+2 K_{N}
$$

has linear growth.

Proposition 5. Let $\Sigma$ be an $H$-surface in $M^{2} \times \mathbf{R}$ satisfying the assumptions of Theorem 3. Then $\int_{\Sigma}|A|^{2}$ has linear growth.

Remark 4. For the proof of this proposition, we do not require that the injectivity radius of $M^{2}$ be bounded below.

Proof. Since we are assuming that $\Sigma$ has finite topology, we may decompose it into a compact piece and a finite number of annular ends. Let $E$ be an annular end of $\Sigma$. We may assume, without loss of generality, that $E$ is a top end, i.e., $h\left(x_{n}\right) \rightarrow \infty$, as $x_{n} \in E$ diverges in $E$, where $h$ is the height function. Then if $h_{\mid \partial E}<0$ and $t \geq 0, E$ transverse to $M_{t}^{2}$, it is clear that $E \cap M_{t}^{2}$ contains at least one Jordan curve $\alpha$ that is a generator of $\pi_{1}(E)$. We call such an $\alpha$ an essential curve. We can 
assume $E \pitchfork M_{0}^{2}$ and (after replacing $E$ by a subend) that $\partial E \subset M_{0}^{2}$. Note that $E$ may intersect $M_{0}^{2}$ in other Jordan curves, but the part of $E$ below height zero is compact and at bounded distance from $M_{0}^{2}$, by our assumption that $h$, restricted to $\Sigma$, is proper.

Let $\phi: \mathbf{S}^{1} \times \mathbf{R}^{+} \rightarrow E$ be a parametrization of $E$ with the property that

$$
\phi\left(\mathbf{S}^{1} \times\{0\}\right)=\partial E \subset M_{0} .
$$

For $a, b \in \mathbf{R}^{+}$with $b>a$, we will assume that $E$ is transverse to $M_{a}^{2}$ and $M_{b}^{2}$, an assumption that is true generically. The set $E_{a}=E \cap M_{a}^{2}$ consists of a finite number of loops, and the same is true of $E_{b}$.

The parametrization $\phi$ orders the essential loops by proximity to $\mathbf{S}^{1} \times\{0\}$ and, in particular, it orders the essential loops in $E_{a}$ and $E_{b}$. Let $\alpha_{1}$ be the first essential loop in $E_{a}$ and let $\beta$ be the first essential loop in $E_{b}$. The annulus $\hat{E}$ bounded by $\alpha_{1}$ and $\beta$ is not necessarily contained in $E_{[a, b]}$. However, by Proposition 11in Section 2 , there exists a constant $C$ (that depends on $H$ and the bounds on the curvature of $M^{2}$, but not on $t$ ) such that regions of $E$ bounded by inessential loops in $M_{t}^{2}$ must lie at a distance of at most $C$ from $M_{t}^{2}$. Hence we may assert that

$$
\hat{E} \subset E_{[a-C, b+C]} .
$$

Using Proposition 1 again, we can assert that if $b-a>2 C$, any essential loop that is past $\beta \subset E_{b}$ in the ordering cannot lie in $E_{a}$. Let $\beta_{1}$ be the last essential loop in $E_{b}$. The loops $\alpha_{1}$ and $\beta_{1}$ bound an annulus $\tilde{E} \subset E$, and we can use Proposition 1 again to assert that

$$
E_{[a+C, b-C]} \subset \tilde{E} \subset E_{[a-C, b+C]} .
$$

Since we are assuming that $\Sigma$ has linear area growth, we know that $E \subset \Sigma$ has linear area growth. This implies that there exists a constant $c$ such that for all $t \geq 0$,

$$
\left|E_{[t, t+1]}\right|<c .
$$

By the co-area formula we know that there exists, for every integer $k \geq 0$, an $a_{k}$, $k \leq a_{k} \leq k+1$, with the property that

$$
\left|E_{a_{k}}\right| \leq c
$$

where $\left|E_{a_{k}}\right|$ is the length of $E_{a_{k}}$. Choose $k_{0}>c+2 C$, let $k>k_{0}+1+2 c+2 C$, and consider $\tilde{E}_{\left[a_{0}, a_{k}\right]}$, the annulus bounded by the essential curves $\alpha_{1} \subset E_{a_{0}}$, and $\beta_{1} \subset E_{a_{k}}$. Choose points $x \in \alpha_{1}$ and $y \in \beta_{1}$, and let $\overline{\alpha_{1}}$ (respectively $\overline{\beta_{1}}$ ) be the minimizing geodesics on $E$ homotopic to $\alpha_{1}$ relative to $x$ (respectively homotopic to $\beta_{1}$ relative to $y$ ). Both $\overline{\alpha_{1}}$ and $\overline{\beta_{1}}$ cannot have length greater than $c$. Let $\bar{E}$ be the annulus in $E$ bounded by $\overline{\alpha_{1}}$ and $\bar{\beta}_{1}$. The exterior angle of $\bar{\alpha}_{1}$ (with respect to $\bar{E})$ at $x$, and the exterior angle of $\bar{\beta}_{1}$ at $y$ cannot be bigger than $\pi$ in absolute value. It is an annulus because we have chosen $k$ large enough to keep $\bar{\alpha}_{1}$ and $\bar{\beta}_{1}$ disjoint. By Gauss-Bonnet, the total curvature of $\bar{E}$ is at most $2 \pi$ in absolute value. By the definition of $\bar{E}$,

$$
E_{*}:=E_{\left[a_{0}+c+C, a_{k}-c-C\right]} \subset \bar{E} \subset E_{\left[a_{k_{0}}-c-C, a_{k}+c+C\right]}=: E^{*} .
$$


Let $c_{0}=\left|4 H^{2}+\sup _{M^{2}} K_{N}\right|$. Using (25), (27) and Lemma 2], we have

$$
\begin{aligned}
\int_{E_{*}}|A|^{2} \leq \int_{\bar{E}}|A|^{2} & =\int_{\bar{E}}\left(4 H^{2}+2 K_{N}\right)-2 \int_{\bar{E}} K \\
& \leq c_{0}|\bar{E}|+4 \pi \\
& \leq c_{0}\left|E^{*}\right|+4 \pi \\
& \leq c_{0} c\left(a_{k}-a_{0}+2(C+c)\right)+4 \pi \\
& \leq c_{0} c(k+1+2(C+c))+4 \pi \\
& \leq \text { constant } \times k,
\end{aligned}
$$

for $k$ large. Hence $\int_{E_{*}}|A|^{2}$ has linear growth, which implies that $\int_{E}|A|^{2}$ also has this property.

Now we present the

Proof of Theorem 3. Because $\Sigma$ has finite topology, it suffices to show that $|A|$ is bounded on each annular end $E$ of $M$. Assume that $E$ is a top annular end of $M$, $\partial E \subset M_{0}=M^{2} \times\{0\}$. Recall that $E_{[a, b]}$ denotes $E \cap\left(M^{2} \times[a, b]\right)$ and $E_{t}=E \cap M_{t}$. We will assume $|A|$ is unbounded on $E$ and obtain a contradiction.

Let $p_{n} \in E$ satisfy $\left|A\left(p_{n}\right)\right| \geq n, n=1,2, \ldots$, and let $p_{n}$ be the lowest point of $E$ with this property. For each integer $n$, let $m=m(n)$ be the smallest integer larger than $h\left(p_{n}\right)+1$. For $q \in E_{[0, m]}$ let $d(q)=m-h(q)$.

Consider the continuous function $F=F_{n}$ defined on $E_{[0, m]}$ :

$$
F(q)=\left\{\begin{array}{l}
|A(q)|, \quad \text { if } \quad h(q) \leq m-1, \\
|A(q)| d(q)^{2}, \quad \text { if } \quad h(q) \geq m-1 .
\end{array}\right.
$$

Let $\tilde{q}=\tilde{q}_{n} \in E$ be a point where $F$ achieves its maximum value. Clearly $|A(\tilde{q})| \geq n$.

Let $a_{n}=h(\tilde{q})-\frac{d(\tilde{q})}{2}, \quad b(n)=h(\tilde{q})+\frac{d(\tilde{q})}{2}$ and consider $\mathcal{E}=\mathcal{E}_{n}=E_{\left[a_{n}, b_{n}\right]}$, the part of $E$ between heights $a_{n}$ and $b_{n}$. If $b_{n} \leq m-1$, then for each $p \in \mathcal{E}$,

$$
|A(p)| \leq|A(\tilde{q})| \text {. }
$$

If $b_{n}>m-1$ and $h(\tilde{q})<m$, then for each $p \in E_{\left[a_{n}, b_{n}\right]}$ we have $d(p) \geq \frac{1}{2}$. So, if $h(p) \geq m$, then $|A(p)| d(p)^{2} \leq|A(\tilde{q})|$, hence

$$
|A(p)| \leq 4|A(\tilde{q})| .
$$

If $h(p) \leq m$, then $|A(p)| \leq|A(\tilde{q})|$. Similarly, the reader can easily check that this inequality also holds if $h(\tilde{q}) \geq m$; so in all cases, when $p \in \mathcal{E}$, one has

$$
|A(p)| \leq 4|A(\tilde{q})| .
$$

At this point, we have produced a sequence $\left\{\tilde{q}_{n}\right\}$ whose heights $h\left(\tilde{q}_{n}\right)$ diverge with $n$, and which have the property that $\left|A\left(\tilde{q}_{n}\right)\right| \geq n$. Moreover, on the subset $\mathcal{E}_{n}=E_{\left[a_{n}, b_{n}\right]} \subset E$, we have the estimate

$$
|A(p)| \leq 4\left|A\left(\tilde{q}_{n}\right)\right| .
$$

Since $p_{n} \in E$ was chosen to be a lowest point where $\left|A\left(p_{n}\right)\right| \geq n$, it follows that $d\left(\tilde{q}_{n}\right) \leq 2$, which in turn implies that $b_{n}-a_{n}=d(\tilde{q}) \leq 2$. Therefore, it follows from 
Proposition 5 that

$$
\int_{\mathcal{E}_{n}}|A|^{2} \leq c
$$

where $c$ does not depend on $n$.

For each $n \geq 0$, translate $E$ down by $h\left(\tilde{q}_{n}\right)$. We may consider $\left\{\tilde{q}_{n}\right\}$ to be a sequence of points in $M_{0}^{2}$, and by abuse of notation, we will refer to these points by the same notation. We also consider the subsets $\mathcal{E}_{n}$ to be similarly translated and we similarly abuse notation. For each $n$, define $\lambda_{n}=\left|A\left(\tilde{q}_{n}\right)\right|$. Rescale the metric on $M^{2} \times \mathbf{R}$ by $\lambda_{n}$ and denote by $\mathcal{E}_{n}^{*}$ the surface $\mathcal{E}_{n}$ in the rescaled metric. The distance between the top and the bottom of $\mathcal{E}_{n}^{*}$ is

$$
\lambda_{n} d\left(\tilde{q}_{n}\right)=\left|A\left(\tilde{q}_{n}\right)\right| d\left(\tilde{q}_{n}\right) \geq n .
$$

We also have from (29) the estimate

$$
\left|A^{*}(p)\right| \leq 4
$$

on $\mathcal{E}_{n}^{*}$ and the normalization $\left|A\left(\tilde{q}_{n}\right)\right|=1$, where $\left|A^{*}\right|$ denotes the second fundamental form in the rescaled metric. The integral in (30) is scale invariant:

$$
\int_{\mathcal{E}_{n}^{*}}\left|A^{*}\right|^{2} \leq c
$$

independent of $n$. Since we are assuming that $\Sigma$ has linear area growth, it follows that $E$ also has linear area growth, and this remains true in the rescaled metrics.

For each $n$, denote by $U_{n}$ the simply connected neighborhood of the origin in $T_{\tilde{q}_{n}}\left(M^{2} \times \mathbf{R}\right)$, which corresponds via the exponential map to $M^{2} \times \mathbf{R}$ minus the cut locus of $\tilde{q}_{n}$. We consider $U_{n}$ with the metric of $M^{2} \times \mathbf{R}$ rescaled by $\lambda_{n}$, and we denote this metric space by $U_{n}^{*}$. Since the injectivity radius of $M^{2}$ is bounded below, and the Gauss curvature of $M^{2}$ is bounded in absolute value, the $U_{n}^{*}$ converge to $\mathbf{R}^{2} \times \mathbf{R}=\mathbf{R}^{3}$ with the flat metric. (The distance from the origin to $\partial U_{n}^{*}$ goes to infinity with $n$ because the of the global lower bound on the injectivity radius and the fact that $\lambda_{n} \rightarrow \infty$. The curvature of $U_{n}^{*}$ behaves like $\frac{\text { const. }}{\lambda_{n}^{2}}$ since there is a global bound on the curvature of $M^{2}$, and hence of $M^{2} \times \mathbf{R}$.)

For each $n$, consider the part of $\mathcal{E}_{n}$ inside the cut locus of $\tilde{q}_{n}^{*}$, and let $\mathcal{E}_{n}^{*}$ be the pullback by the exponential map to $U_{n}^{*}$ of the component of this surface containing the point $\tilde{q}_{n}^{*}$. The mean curvature of $\mathcal{E}_{n}^{*}$ is $\frac{H}{\lambda_{n}}$, which goes to zero as $n \rightarrow \infty$. We have established in (31) bounds for the integral of $\left|A^{*}\right|^{2}$. We are assuming linear growth for $\Sigma$, and this implies that $\mathcal{E}_{n}^{*}$ has linear area growth. Therefore, we may choose a subsequence of the $\mathcal{E}_{n}^{*}$ which converges - with finite multiplicity - to an embedded minimal surface, $\mathcal{S}$, in $\mathbf{R}^{3}$ with the flat metric. Moreover, $\mathcal{S}$ has $\left|A^{*}(0)\right|=1$ and $\int_{\mathcal{S}}\left|A^{*}\right|^{2}$ is finite by (31). Since $|A|^{2}=-2 K, \mathcal{S}$ has finite total curvature, but is not the plane because $\left|A^{*}(0)\right| \neq 0$. It is complete because $\Sigma$ was complete, and the distance from the origin to the boundary of $\Sigma_{n}^{*}$ is not less than the distance from the origin to the boundary of $U_{n}^{*}$, which diverges with $n$.

In the previous paragraph we have shown that $\mathcal{S} \subset \mathbf{R}^{3}$ is a complete and nonplanar embedded minimal surface with finite total curvature. Such a surface must have a top and a bottom catenoidal end. Consider a top catenoidal end $\bar{\Sigma}$ of $\mathcal{S}$. Let $\gamma$ be a Jordan curve on $\bar{\Sigma}$, which is the tranverse intersection of $\Sigma$ with some plane orthogonal to the axis of the top catenoid end. The flux of $\bar{\Sigma}$ along $\gamma$ is a vector 
whose component, $a$, in the direction of the axis of the catenoidal end, is equal to the logarithmic growth of the end. Since the end is catenoidal, $a \neq 0$.

Now $k \gamma=\lim \gamma_{n}$, where $\gamma_{n}$ are Jordan curves on $\Sigma_{n}^{*}$ and $k$ is the (fixed) multiplicity with which the $\mathcal{E}_{n}^{*}$ (a subsequence) are converging to $\mathcal{S}$. The $\gamma_{n}$ are coming from loops $\beta_{n}$ on $E \subset \Sigma$ by scaling by $\lambda_{n}$ with $\lambda_{n} \rightarrow \infty$. We know that this flux is zero if $\beta_{n}$ is homologous to zero on $E$ and equals some fixed vector when $\beta_{n}$ is a generator of $\pi_{1}(E)$. Let $b$ be the component of this vector in the direction determined by the top catenoid end. Then one has $\lambda_{n} b \rightarrow k a$, as $\lambda_{n} \rightarrow \infty$. Since $b$ is finite, this is a contradiction.

Remark 5. We conjecture that a properly embedded $H$-surface, $\Sigma$, of finite topology in $M^{2} \times \mathbf{R}$ must have linear area growth if $M^{2}$ is compact. (This is true when $H=0$, 16].) Using Theorem 3 and arguing as in Corollary 2, this conjecture, if true, implies that such a $\Sigma$ would have bounded second fundamental form.

\section{REFERENCES}

[1] J. L. Barbosa and R.S. Earp. Prescribed mean curvature in $H^{n+1}(-1)$ with convex planar boundary, I. Geom. Dedicata, 71(1):61-74, 1998. MR.1624726 (99d:53064)

[2] M. Crandall and P. Rabinowitz. Bifurcation, perturbation of simple eigenvalues and linearized stability. Arch. Rational Mech. Anal., 52:161-180, 1973. MR0341212 (49:5962)

[3] P. Collin. Topologie et courboure des surfaces minimales proprement plongées de $\mathbf{R}^{3}$. Ann. of Mathematics, 145:1-31, 1997. MR1432035 (98d:53010)

[4] D. Gilbarg and N. S. Trudinger. Elliptic partial differential equations of second order. Springer-Verlag, New York, 2nd edition, 1983. MR0737190 (86c:35035)

[5] Karsten Grosse-Braukmann and Meinhard Wohlgemuth. The gyroid is embedded and has constant mean curvature companions. Calc. Var., 4:499-523, 1996. MR.1415998 (97k:53011)

[6] D. Hoffman and H. Karcher. Complete embedded minimal surfaces of finite total curvature. In Encyclopedia of Mathematics, pages 5-93, 1997. R. Osserman, editor, Springer Verlag. MR1490038 (98m:53012)

[7] D. Hoffman and W. H. Meeks III. Embedded minimal surfaces of finite topology. Annals of Mathematics, 131:1-34, 1990. MR1038356 (91i:53010)

[8] W.-T. Hsiang and W.-Y. Hsiang. On the uniqueness of isoperimetric solutions and imbedded soap bubbles in non-compact symmetric spaces. Inventiones Math. 98:39-58, 1989. MR1010154 (90h:53078)

[9] H. Karcher. Embedded minimal surfaces derived from Scherk's examples. Manuscripta Math., 62:83-114, 1988. MR0958255(89i:53009)

[10] H. Karcher. The triply periodic minimal surfaces of Alan Schoen and their constant mean curvature companions. Manuscripta Math., 64:291-357, 1989. MR.1003093 (90g:53010)

[11] N. Korevaar, R. Kusner, and B. Solomon. The structure of complete embedded surfaces with constant mean curvature. Journal of Differential Geometry, 30:465-503, 1989. MR 1010168 (90g:53011)

[12] H. B. Lawson. Complete minimal surfaces in $S^{3}$. Annals of Mathematics, 92:335-374, 1970. MR0270280 (42:5170)

[13] H. B. Lawson, Jr. Lectures on Minimal Submanifolds. Publish or Perish Press, Berkeley, 1971. MR 0576752 (82d:53035b)

[14] R. Mazzeo and F. Pacard. Bifurcating nodoids. Contemp. Math., 314:169-186, 2002. MR.1941630 (2004b:53015)

[15] W. H. Meeks III and H. Rosenberg. The uniqueness of the helicoid. Annals of Mathematics 161(2):723-754, 2005. MR2153399

[16] W. H. Meeks III and H. Rosenberg The theory of minimal surfaces in $M \times \mathbf{R}$. To appear in Comment. Math. Helv.

[17] B. Nelli and H. Rosenberg. Minimal surfaces in $\mathbf{H}^{2} \times \mathbf{R}$. Bull. Bras. Math. Soc., New Series, 33(2):263-292, 2002. MR1940353 (2004d:53014)

[18] B. Nelli and H. Rosenberg. Global Properties of Constant Mean Curvature Surfaces in $\mathbf{H}^{2} \times \mathbf{R}$. To appear in Pacific J. of Mathematics. 
[19] P. A. Nitsche. Existence of prescribed mean curvature graphs in hyperbolic space. Manuscripta Math., 108(3):349-367, 2002. MR1918082 (2003f:53015)

[20] R. Pedrosa and M. Ritoré. Isoperimetric domains in the Riemannian product of a circle with simply connected space forms and applications to free boundary problems. Indiana Univ. J. of Mathematics, 48:1357-1394, 1999. MR:1757077 (2001k:53120)

[21] H. Rosenberg. Minimal surfaces in $M^{2} \times \mathbf{R}$. Illinois J. Math, 46(4):1177-1195, 2002. MR1988257 (2004d:53015)

[22] H. Rosenberg. Hypersurfaces of constant curvature in space forms. Bull Sc. Math, 117:211239, 1993. MR1216008 (94b:53097)

[23] R. Schoen. Analytic aspects of the harmonic map problem. In Seminar on Nonlinear Partial Differential Equations, 1983. MR0765241 (86b:58032)

[24] J. Serrin. The problem of Dirichlet for quasilinear elliptic differential equations with many independent variables. Philos. Trans. Roy. Soc. London Ser. A, 264:413-496, 1969. MR0282058 $(43: 7772)$

[25] J. Smoller. Shock waves and reaction-diffusion equations. Springer-Verlag, New York, 1983. MR0688146 (84d:35002)

[26] M. Weber and M. Wolf. Teichmüller theory and handle addition for minimal surfaces. Annals of Math. 156:713-795, 2002. MR1954234 (2005j:53012)

[27] M. Weber and M. Wolf. Minimal surfaces of least total curvature and moduli spaces of plane polygonal arcs. GAFA, 8:1129-1170, 1998. MR1664793 (99m:53020)

[28] M. Wohlgemuth. Higher genus minimal surfaces by growing handles out of a catenoid. Manuscripta Math., 70:397-428, 1991. MR1092145(91k:53021)

Department of Mathematics, Stanford University, Stanford, California 94305

E-mail address: hoffman@math.stanford.edu

Departamento de Matemática, Universidade Federal do Ceará, Fortaleza - Ceará BRASIL

E-mail address: jherbert@mat.ufc.br

Institut de Mathematiques de Jussieu, Paris XIII, France

E-mail address: rosen@math.jussieu.fr 